\title{
Efectos del entrenamiento con movimientos de halterofilia en el rendimiento de esprint, salto y cambio de dirección en deportistas: Una revisión sistemática Effects of weightlifting training on sprint, jump and change of direction performance in athletes: A systematic review \\ *Cristián Mateluna-Núñez, *Juan Pablo Zavala-Crichton, **Matías Monsalves-Álvarez, ***Jorge Olivares-Arancibia, ****Rodrigo Yáñez-Sepúlveda \\ *Universidad Andres Bello (Chile); **Universidad de O’Higgins, Rancagua (Chile), **Motion Health \& Performance Center (Chile); ***Universidad de las Américas (Chile); ****Universidad Viña del Mar (Chile)
}

Resumen: La capacidad de generar máxima potencia neuromuscular es el factor más importante y determinante en el rendimiento atlético. Debido a esto, el entrenamiento con movimientos de Halterofilia (EMH) y sus derivados es uno de los métodos más usados, ya que la evidencia muestra que genera adaptaciones de fuerza-potencia superiores comparadas con el entrenamiento de fuerza tradicional, de salto y de kettlebells. Objetivo: Identificar los efectos del EMH en la capacidad de salto, esprint y cambio de dirección (COD) en población deportista. Método: Se realizó una búsqueda exhaustiva en diferentes bases de datos, como PUBMED, Sportdiscus (EBSCO), Scopus y Web of Science (WOS) bajo modelo PRISMA. Los trabajos revisados fueron experimentales con y sin grupo de control, entre los años 2000 y 2020 . Resultados: El EMH produce mejoras significativas en las capacidades de salto, de esprint y de COD en población deportista. Conclusión: El EMH genera mejoras significativas en el rendimiento de salto, carreras y cambio de dirección bajo distintos protocolos. Existe evidencia que sustenta la aplicación de EMH, recomendando sus derivados centrados en el segundo tirón y aquellos que utilicen el ciclo de estiramiento-acortamiento en sus variantes colgantes.

Palabras claves: Entrenamiento de fuerza, Rendimiento deportivo, ejercicios derivados de la halterofilia, Entrenamiento de potencia, Tasa de desarrollo de fuerza.

\begin{abstract}
The ability to generate maximum power is the most important and determining neuromuscular function in sports performance. Therefore, weightlifting training (WT) and its derivatives is one of the most widely used methods, generating superior strength-power adaptations compared to traditional strength training, jumping and kettlebell training. Objective: To identify the effects of WT on the ability to jump, sprint and change of direction (COD) in athletes. Method: An exhaustive search was carried out in different databases, such as PUBMED, Sportdiscus (EBSCO), Scopus and Web of Science (WOS) under the PRISMA model. The reviewed papers were experimental with and without a control group, between the years 2000 and 2020. Results: The WT produces significant improvements in jump, sprint and in change of direction capacities in the sport population. Conclusion: WT generates significant improvements in jumping, running and change of direction performance under different protocols. There is evidence supporting the use ofWT, suggesting its derivatives focused on the second pull and those that use the stretch-shortening cycle in their hanging variants.
\end{abstract}

Keywords: Strength training, Sport performance, Weightlifting derivatives, Power training, Rate force of development.

\section{Introducción}

La capacidad de generar máxima potencia neuromuscular es el factor más importante y determinante en el rendimiento deportivo (Cormie, McGuigan, \& Newton, 2011; Kawamori \& Haff, 2004; Holmberg, 2013; Schilling, 2016). Debido a esto, las alternativas metodológicas para entrenar esta capacidad son foco de estudio en las ciencias del deporte, y una de ellas es el entrenamiento con movimientos de halterofilia (EMH). La halterofilia presenta dos movimientos competitivos,

Fecha recepción: 01-04-21. Fecha de aceptación: 11-11-21

Cristián Andrés Mateluna Núñez

matelunanc2@gmail.com la Arrancada (Snatch) que busca llevar la barra desde la plataforma hasta la completa extensión de los brazos sobre la cabeza y la cargada y envión (Clean \&Jerk), que tiene el mismo objetivo, pero a través de dos movimientos. (International Weightlifting Federation, 2018). Como método, el EMH y sus derivados es uno de los más usados por generar adaptaciones de fuerza-potencia superiores (Suchomel, Nimphius, Bellon, \& Stone, 2018) al entrenamiento de fuerza tradicional (RTT) (Hoffman, Cooper, Wendell, \& Kang, 2004; Channell \& Barfield, 2008; Chaouachi et al., 2014; Arabatzi \& Kellis, 2012), al entrenamiento con saltos (Tricoli, Lamas, Carnevale, \& Ugrinowitsch, 2005; Teo, Newton, Newton, Dempsey, \& Fairchild, 2016), y al entrena- 
miento con kettlebells (Otto, Coburn, Brown, \& Spiering, 2012). En consecuencia, estos movimientos han sido utilizados para el acondicionamiento físico de muchos deportes (Chiu \& Schilling, 2005; Simenz, Dugan, \& Ebben, 2005; Ebben, Carrol, \& Simenz, 2004; Ebben, Hintz, \& Simenz, 2005), ya que desarrollan la fuerza del tren inferior mejorando el desempeño potente en cualquier tarea deportiva que requiera una triple extensión, (cadera, rodilla y tobillo) como lo son el salto, el esprint y los cambios de dirección (COD) (Suchomel, Comfort, \& Stone, 2015).

Los movimientos de Halterofilia al ser un deporte y un método de desarrollo de fuerza- potencia eficaz (Chiu \& Schilling, 2005), se utilizan respondiendo a la especificidad de otras actividades competitivas por ejemplo, a través de movimientos derivados que se centran en la ejecución de ciertas fases específicas del gesto para una aplicación más segura y eficiente (Suchomel et al., 2015; Soriano, Suchomel, \& Comfort, 2019). Un grupo de estos ejercicios son los tirones derivados de la arrancada y la cargada (TDH) (DeWeese et al., 2016; Suchomel, DeWeese, Beckham, Serrano, \& Sole, 2014; Suchomel, DeWeese, Beckham, Serrano, \& French, 2014; DeWeese, Serrano, Scruggs, \& Burton, 2013; DeWeese, Serrano, Scruggs, \& Sams, 2012; DeWeese \& Scruggs, 2012), que se implementan omitiendo la fase técnica de recepción de la barra y centrándose únicamente en el tirón (Suchomel et al., 2015). En estudios previos, se observa que en la realización de TDH, como el tirón de cargada y arrancada (Clean pull, Snatch pull), el tirón de cargada y arrancada colgante (Hang clean pull, Hang Snatch pull), los tirones altos de cargada y arrancada (High pull), y el salto con encogimiento de hombros (Jump Shrug), se alcanzan mayores tasas de producción de fuerza (RFD), fuerza máxima dinámica, velocidad y potencia, en comparación con ejercicios de halterofilia que no omitan la fase de recepción como la arrancada y cargada de potencia (Power clean) (Suchomel et al., 2015; Comfort, Allen, \& Graham-Smith, 2011a; Comfort, Allen, \& Graham-Smith, 2011b; Suchomel, Wright, Kernozek, \& Kline, 2014). Adicionalmente, la implementación de los TDH permiten el uso eficiente del tiempo de entrenamiento en base a la disminución de su complejidad de enseñanza (Hedrick, 2004; Janz, Dietz, \& Malone, 2008; Harbili \& Alptekin, 2014), reducción en los impactos que permiten evitar lesiones (Kulund, Dewey, Brubaker, \& Roberts, 1978; Stone, Fry, \& Ritchie, 1994; Suchomel \& Sato, 2013), generar adaptaciones con cargas superiores a una repetición máxima (1RM) (Comfort, Udall, \& Jones, 2012), y su aplicación como complejos potenciadores balísticos (Chiu \& Salem, 2012; Maloney, Turner, \& Fletcher, 2014). Por otra parte, los empujes derivados de la halterofilia (EDH) tales como el Push press, Push jerk o Split jerk, se desarrollan verticalmente y el movimiento finaliza con los hombros flexionados y los codos totalmente extendidos por encima de la cabeza. Estos ejercicios podrían ser beneficiosos para la mejora de la habilidad de desarrollar altos niveles de fuerza rápida, debido a la potente triple extensión de caderas, rodillas y tobillos que es típica de la fase de empuje y que además, es similar a otras tareas deportivas (Cushion, Goodwin, \& Cleather, 2015).Adicionalmente, los EDH pueden considerarse un estímulo variado que demanda dominio técnico, trabajo de equilibrio y coordinación (Soriano et al., 2019).

Otro elemento relevante en la habilidad de generar máxima fuerza es la capacidad de aplicarla en el menor tiempo posible. Esta variable se evalúa en la RFD, la cual parece estar determinada principalmente por el factor neural (Maffiuletti et al., 2016) y tiene una marcada implicación en el ámbito deportivo, puesto que en la competición se presentan muchas situaciones donde el tiempo para aplicar la fuerza es limitado (RodríguezRosell, Pareja-Blanco, Aagaard, \& González-Badillo, 2018). Por lo tanto, cualquier entrenamiento que conlleve la realización de esfuerzos balísticos, es decir, que exijan al atleta acelerar durante todo el rango de movimiento, incrementará la producción de fuerza y puede generar mejoras en la RFD (Suchomel, Nimphius, \& Stone, 2016; Cormie et al., 2011). Por esta razón, los TDH pueden ser herramientas de entrenamiento adecuadas, ya que en ellos se combinan componentes de fuerza y velocidad, permitiendo mover cargas moderadas y pesadas a velocidades relativamente altas, manteniendo la intención balística del movimiento (Suchomel \& Sole, 2017).

En cuanto a la capacidad de salto, diversas revisiones han dejado de manifiesto que el EMH es más eficaz para mejorar el rendimiento en salto comparado al entrenamiento de fuerza tradicional, y genera resultados similares en comparación con el entrenamiento pliométrico (Berton, Lixandrão, Pinto e Silva, \& Tricoli, 2018; Hackett, Davies, Soomro, \& Halaki, 2016). Con relación al rendimiento de esprint, desde la revisión de Hedrick (2018) se evidencia que el EMH ha mostrado mejoras en rugbistas y hombres entrenados, particularmente en distancias de 10 metros. Junto con ello, al analizar las prácticas habituales y las recomendaciones con base científica, se sugiere el EMH para la mejora en el esprint (Haugen, Seiler, Sandbakk, \& Tønnessen, 
2019). En relación a los COD, se mencionó anteriormente que el EMH influye positivamente en las tareas deportivas que impliquen una triple extensión (Suchomel et al., 2015), sin embargo, desde la revisión de Brughelli, Cronin, Levin, \& Chaouachi (2008) se concluyó que el RTT y el EMH en su mayoría no lograron mejoras en esta habilidad, a diferencia del entrenamiento de COD específico y general.

Considerando que existe una amplia variedad de ejercicios de Halterofilia (Suchomel et al., 2015; Soriano et al., 2019), y que la mayoría de la revisiones anteriores se centran en diversas poblaciones, el propósito de esta revisión es identificar los efectos del EMH en la capacidad de salto, de esprint y COD únicamente en población deportista. minos (p. ej. Weightlifting training (EMH) OR Weightlifting variations), y AND para una búsqueda más restringida que cruza 2 o más términos (p. ej. Weightlifting training (EMH) AND Jump performance) (Trueba-Gómez \& Estrada-Lorenzo, 2010). En cada base de datos se buscó en los campos de título, resumen y el de palabras claves.

La estrategia de búsqueda en la primera fase consistió en la identificación en las bases de datos y posteriormente la eliminación de duplicados. A continuación, en la fase de screening se realizó el filtrado de artículos por títulos, resúmenes y la eliminación por criterios de inclusión. Finalmente, en la fase 3 se realizó lectura y análisis de forma íntegra de todos los artículos que entraron a revisión (Figura 1).

\section{Método}

\section{Búsqueda bibliográfica}

Se realizó una búsqueda electrónica exhaustiva en las siguientes bases de datos: PUBMED, Sportdiscus (EBSCO), Scopus y Web of Science (WOS). Los límites de búsqueda consideraron publicaciones comprendidas entre los años 2000 a 2020 y sin limitadores de idioma. La selección y análisis de artículos científicos se realizó en concordancia a la guía de publicación de revisiones sistemáticas y meta-análisis PRISMA (Hutton, Catalá-López, \& Moher, 2016). Las palabras clave utilizadas para la revisión fueron combinadas con los operadores booleanos OR para un conjunto de 2 o más tér-

\begin{tabular}{|c|c|c|c|c|c|}
\hline \multicolumn{6}{|c|}{ Estrategias de búsqueda } \\
\hline Pasos & Estrategia & PUBMED & SPORTDISCUS & SCOPUS & WOS \\
\hline 1 & Weightlifting & 855 & 1882 & 817 & 742 \\
\hline 2 & Weightlifting training & 456 & 61 & 412 & 374 \\
\hline 3 & Weightlifting variations & 49 & 4 & 54 & 55 \\
\hline 4 & Weightlifting derivatives & 43 & 18 & 37 & 31 \\
\hline 5 & Snatch & 317 & 591 & 368 & 717 \\
\hline 6 & Clean \& Jerk & 52 & 42 & 91 & 90 \\
\hline 7 & Power clean Weightlifting & 64 & 1 & 84 & 105 \\
\hline 8 & Hang power clean & 38 & 18 & 39 & 51 \\
\hline 9 & Elean pull & 150 & 36 & 238 & 456 \\
\hline 10 & Snatch pull & 33 & 12 & 48 & 71 \\
\hline 11 & Hang high pull & 19 & 9 & 25 & 64 \\
\hline 12 & Jump Shrug & 10 & 8 & 10 & 10 \\
\hline 13 & Mid-thigh pull & 71 & 45 & 74 & 72 \\
\hline 14 & Standing press & 650 & 37 & 3614 & 1512 \\
\hline 15 & Push press & 274 & 59 & 1768 & 783 \\
\hline 16 & Push Jerk & 11 & 3 & 15 & 18 \\
\hline 17 & Split Jerk & 6 & 1 & 40 & 40 \\
\hline \multirow[t]{4}{*}{18} & \#1 OR \#2 OR \#3 \#OR 4 OR \#5 & 1242 & 2534 & 6763 & 4168 \\
\hline & OR \#6 OR \#7OR \#8 OR \#9 OR & & & & \\
\hline & \#10 OR \#11 OR \#12 OR \#13 OR & & & & \\
\hline & \#14 OR \#15 OR \#16 OR \#17 & & & & \\
\hline 19 & Athletic performance & 43456 & 2211 & 17869 & 7075 \\
\hline 20 & Sport performance & 58430 & 6393 & 35438 & 30239 \\
\hline 21 & Sprint performance & 5694 & 1305 & 5503 & 7314 \\
\hline 22 & Change of direction performance & 26279 & 186 & 10235 & 11062 \\
\hline 23 & Jump performance & 6900 & 1365 & 10488 & 13108 \\
\hline 24 & $\# 19 \# 20 \# 21 \# 22 \# 23$ & 140759 & 10282 & 60456 & 57177 \\
\hline 25 & \#18 AND \#21 & 46 & 32 & 63 & 94 \\
\hline 26 & \#18 AND \#22 & 26 & 11 & 23 & 21 \\
\hline 27 & \#18 AND \#23 & 152 & 64 & 171 & 265 \\
\hline
\end{tabular}

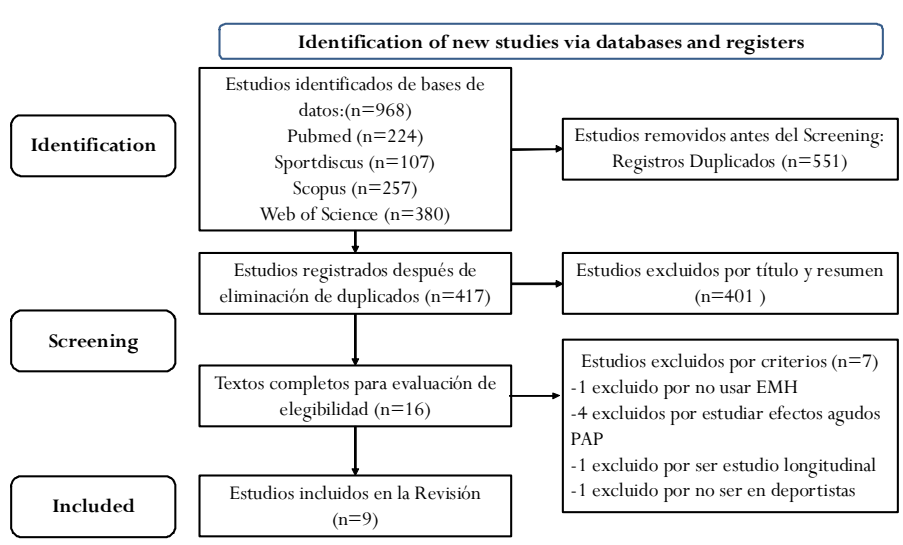

Figura 1. Diagrama de flujo PRISMA

\section{Criterios de inclusión y exclusión}

Para la elegibilidad de los estudios, se consideraron los criterios que se presentan en la Tabla 2, los cuales fueron construidos a través de la herramienta PICOS (Methley, Campbell, Chew-Graham, McNally, \& Cheraghi-Sohi, 2014). En cuanto a la población, se excluye a weightlifters (deportistas de Halterofilia) porque el EMH es prácticamente la realización de sus movimientos de competición, y en contra partida se considera en la inclusión a deportistas adultos y adolescentes, ya sea profesionales, universitarios o de competencias nacionales y regionales.

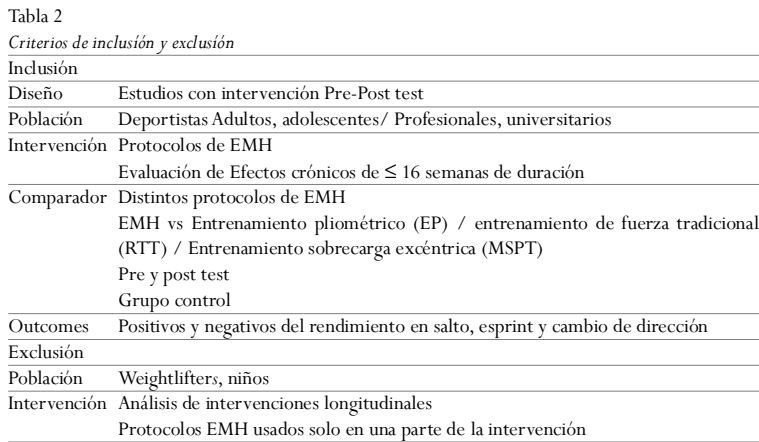




\section{Evaluación de la calidad metodológica}

Para evaluar la calidad metodológica de los estudios se utilizó la escala Physiotherapy Evidence Database (PEDro) (de Morton, 2009; Herbert, Moseley, Sherrington, \& Maher, 2000). La clasificación se realizó en base a tres criterios: selección (máximo tres puntos), comparabilidad (máximo tres puntos) y resultados (máximo cuatro puntos). Descrito en la Tabla 3, los artículos con puntuación de ocho a diez se consideraron de calidad metodológica alta, los puntuados de cuatro a siete de calidad moderada y los de puntuación menor de cuatro de calidad baja. La puntuación obtenida por los artículos según la escala PEDro indica que los nueve artículos registraron una puntuación entre cuatro a siete, obteniendo una calidad metodológica moderada.

Tabla 3

\begin{tabular}{|c|c|c|c|c|c|c|c|c|c|c|c|c|}
\hline \multirow{3}{*}{ Estudios } & \multicolumn{12}{|c|}{ Criterios de calidad PEDro } \\
\hline & \multicolumn{4}{|c|}{ Selección } & \multicolumn{3}{|c|}{ Comparabilidad } & \multicolumn{4}{|c|}{ Resultados } & \multirow[b]{2}{*}{ Pts. } \\
\hline & 1 & 2 & 3 & 4 & 5 & 6 & 7 & 8 & 9 & 10 & 11 & \\
\hline Avers et. al, 2016 & Sí & Sí & No & Sí & No & No & No & Sí & Sí & Sí & Sí & 6 \\
\hline Ciacci \& Bartolomei & Sí & Sí & No & Sí & No & No & No & Sí & Sí & Sí & Sí & 6 \\
\hline Comfort et. al, 2018 & Sí & No & No & Sí & No & No & No & No & Sí & Sí & Sí & 4 \\
\hline Helland et. al, 2017 & Sí & Sí & No & Sí & No & No & No & No & Sí & Sí & Sí & 5 \\
\hline HermasSí et. al, 2019 & Sí & Sí & No & Sí & No & No & No & Sí & Sí & Sí & Sí & 6 \\
\hline Ince, 2019 & Sí & Sí & No & Sí & No & No & No & Sí & Sí & Sí & Sí & 6 \\
\hline Keller et. al, 2018 & Sí & Sí & No & Sí & No & No & No & Sí & Sí & Sí & Sí & 6 \\
\hline Loturco et. al, 2016 & Sí & Sí & No & Sí & No & No & No & No & Sí & Sí & Sí & 5 \\
\hline Oranchuk et al, 2019 & Sí & Sí & No & Sí & No & No & No & Sí & $S_{i ́}^{\prime}$ & Sí & Sí & 6 \\
\hline
\end{tabular}

\section{Procedimientos de evaluación}

\section{Rendimiento del salto}

Para evaluar el rendimiento de la saltabilidad, los estudios consideraron el salto con contramovimiento $(\mathrm{CMJ})$, salto sin contramovimiento (SJ) y salto contramovimiento con ayuda de brazos (CMJbra) que miden la expresión de la fuerza explosiva (potencia) del salto vertical (Bosco, Luhtanen, \& Komi, 1983). Mientras que el SJ busca evaluar la fuerza explosiva de la acción concéntrica y la coordinación intra e intermuscular, el CMJ evalúa además la reutilización de la energía elástica (Bosco, 1994). Al igual que el CMJ, el CMJbra mide la reutilización elástica, pero sumando la potenciación del impulso de los brazos que aumenta la necesidad de coordinación dinámica general. Es interesante destacar que el CMJ, además de evaluar la potencia puede servir como instrumento de control de la fatiga neuromuscular, ya sea de forma aguda o a lo largo de una temporada (Miras, 2019).

El salto horizontal (SLJ) representa un índice general de fuerza muscular en la juventud, por tener una alta correlación con otras pruebas de fuerza muscular de la parte superior e inferior del cuerpo (Castro-Piñero et al., 2010). De la misma manera, el salto lateral de 1 pie (1LLJ) tiene una alta correlación con el rendimien- to de sprint de 10 metros en hombres (Meylan et al., 2009), ambos saltos, SLJ y 1LLJ miden la fuerza en el vector horizontal. También se midió el Spike jump, un salto de ataque que responde a las necesidades de evaluar expresiones de fuerza específicas del vóleibol, y que tiene una alta confiabilidad y validez comparadas con las pruebas clásicas de CMJ y SJ (Sattler, Sekulic, Hadzic, Uljevic, \& Dervisevic, 2012). En definitiva, la utilización de distintos saltos permite evaluar la potencia del tren inferior, basado en que la potencia muscular se relaciona con el rendimiento físico y deportivo (García-Chaves, Corredor-Serrano, \& Arboleda-Franco, 2020).

\section{Rendimiento de Sprint}

Las pruebas de esprint, consideraron distancias de 5 , $10,15,20,30$ metros y 40 yardas $(36,5 \mathrm{~m})$, en este sentido, la variedad de distancias se explica porque los recorridos son distintos en cada deporte (Sweeting et al., 2017). De hecho, la variedad de distancias recorridas es tan amplia que incluso puede diferir en un mismo deporte, ya sea por sexo o por nivel, como se evidenció en basquetbolistas de nivel nacional y estatal en el estudio de Scanlan, Dascombe, \& Reaburn, (2011). Además de los recorridos, la variedad de pruebas de esprint es debido a que tampoco existe claridad en los umbrales de velocidad y aceleración, encontrando por ejemplo, distintos umbrales de alta velocidad para el fútbol australiano ya sea en $>4.00 \mathrm{~m} / \mathrm{s}$ (Sullivan et al., 2014) o en $>4.17 \mathrm{~m} / \mathrm{s}$ (Mooney et al., 2011). Esta variedad de pruebas, fruto de la dificultad para determinar cuando un esprint es un esprint, impacta también en tener que entrenar esta capacidad desde un enfoque más holístico (Hori et al., 2008).

\section{Rendimiento del cambio de dirección}

Existen variadas pruebas para evaluar el rendimiento del COD, que pueden clasificarse por sistema energético, tipo de aplicación de fuerza y número de cambios de dirección (Brughelli et al., 2008). En cuatro estudios de esta revisión, se reconocieron evaluaciones con distintos números de COD (p. ej. 6COD test y 13COD test) lo que, junto a la longitud de las pruebas, influyen en su duración y por tanto en los sistemas energéticos predominantes. En este sentido, pruebas que duren hasta los cinco segundos tendrán un mayor aporte de fosfágenos, y las con mayor duración comenzarán a recibir energía de vías glucolíticas y posteriormente aeróbicas (Gastin, 2001). En relación a la clasificación por tipo de aplicación de la fuerza, en los T test de estos 
estudios encontramos aplicaciones de fuerza horizontal (Gabbett et al., 2006), T test con aplicación de fuerza horizontal y lateral (Mcbride et al., 2002), yT test modificado con las mismas aplicaciones de fuerzas (Cronin, McNair, \& Marshall, 2003).

\section{Resultados}

Una vez realizada la búsqueda bibliográfica, se obtuvieron 968 estudios que luego de verificación de duplicación quedaron en 417. Posteriormente, en la exclusión por título y resumen se obtuvieron 16 estudios que pasaron a lectura completa, dando como resultado nueve estudios incluidos en la revisión. De los estudios incluidos que informaban los efectos EMH, nueve midieron sus efectos en la capacidad de salto, cinco en la capacidad de esprint y cuatro en la capacidad de COD.

\section{Efectos del EMH en el rendi- miento de salto}

Como se aprecia en la tabla 4, en el primer estudio de Ayers et al. (2016), las dos intervenciones basadas en Hang clean y Hang Snatch mostraron mejoras significativas en $\mathrm{CMJ}$ pre y post intervención $(p \leq 0,01)$.

Para el estudio de Ciacci \& Bartolomei (2018) realizado en basquetbolistas, las categorías Senior y U19 lograron mejoras significativas $(p<0,05)$ en todas las variables (SJ, CMJ, CMJbra, CMJ1p) y en los dos protocolos (EMH y RTT), mientras que el grupo de U17 con el protocolo RTT mejoró en SJ y en CMJ1p. El estudio de Comfort et al. (2018), que comparó ejercicios de Power clean con y sin fase de captura (catch/pull) mostró mejoras en sus dos intervenciones en CMJ $(p<0,001)$, sin tener diferencias significativas entre ambas. También Hermassi et al. (2019) evidenció mejoras significativas $(p<$ 0,001) en SJ y CMJ.

Por otro lado, en el estudio comparativo de protocolos de Helland et al. (2017), el EMH obtuvo mejoras triviales en SJ, CMJ, DJ, y además menores en comparación con los protocolos de sobrecarga excéntrica (MSPT) y peso libre (FSPT). Por su parte, los protocolos de Loturco et al. (2016) e Ince (2019), ambos de 6 semanas no lograron mejoras significativas en CMJ, sin embargo, este último generó mejoras significativas en Spike jump que corresponde a un salto deportivo específico del voleibol. También, el protocolo de entrenamiento explosivo para basquetbolistas de Keller et al. (2018) mostró mejoras significativas ( $p$ $<0,05)$ en CMJ, SLJ, 1LLJ, al igual que el protocolo de Oranchuk et al. (2019) que mejoró SJ y CMJ.

Efectos del EMH en el rendimiento de esprint Como se aprecia en la tabla 5, en el estudio de Ayers
Tabla 4

Efectos de EMH en salto

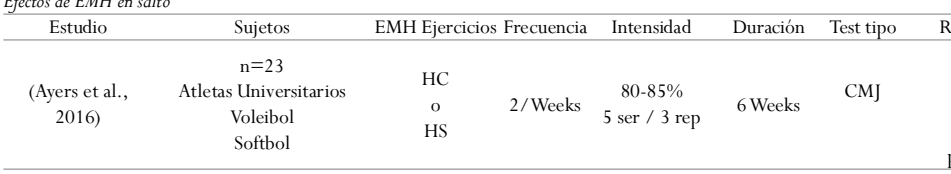

\begin{tabular}{|c|c|c|c|c|c|c|c|}
\hline $\begin{array}{c}\text { (Ciacci \& } \\
\text { Bartolomei, 2018) }\end{array}$ & $\begin{array}{c}\mathrm{n}=58 \\
\text { Baloncesto } \\
\text { Nivel nacional } \\
\text { Senior } \\
\text { U19 } \\
\text { U17 }\end{array}$ & $\begin{array}{c}\mathrm{PC}+\text { rope } \\
\quad \mathrm{o} \\
\mathrm{HSQ}+\text { escalera }\end{array}$ & 2/Weeks & $\begin{array}{c}4 \text { ser/ } 8 \text { rep } \\
50 \%\end{array}$ & 16 Weeks & $\begin{array}{c}\text { SJ } \\
\text { CMJ } \\
\text { CMJbra } \\
\text { CMJ1p }\end{array}$ & $\begin{array}{c}\text { Senior/U19 } \\
\text { HC } \\
\uparrow \mathrm{SJ} \uparrow \mathrm{CMJ} \\
\uparrow \mathrm{CMJbra} \\
\uparrow \mathrm{CMJ} 1 \mathrm{p} \\
\text { Senior HSQ } \\
\uparrow \mathrm{SJ} \uparrow \mathrm{CMJ} \\
\uparrow \mathrm{CMJbra} \\
\uparrow \mathrm{CMJ} 1 \mathrm{p} \\
\mathrm{U} 19 \mathrm{HSQ} \\
\uparrow \mathrm{SJ} \uparrow \mathrm{CMJ} 1 \mathrm{p} \\
\mathrm{U} 17 \mathrm{HSQ} \\
\uparrow \mathrm{SJ} \uparrow \mathrm{CMJ} 1 \mathrm{p} \\
\mathrm{p}<0,05\end{array}$ \\
\hline $\begin{array}{c}\text { (Comfort et al., } \\
\text { 2018) }\end{array}$ & $\begin{array}{c}\mathrm{n}=11 \\
\text { Futbolistas juveniles } \\
\text { profesionales } \\
\mathrm{n}=23 \\
\text { Atletas universitarios } \\
\text { (BMX, Remo Hockey) }\end{array}$ & $\begin{array}{c}\text { PC Catch } \\
\text { o } \\
\text { PC Pull }\end{array}$ & 2/Weeks & $\begin{array}{l}3 \times 5 / 75 \% \\
3 \times 5 / 80 \% \\
3 \times 5 / 82.5 \% \\
3 \times 5 / 67.5 \% \\
3 \times 3 / 80 \% \\
3 \times 3 / 85 \% \\
3 \times 3 / 90 \% \\
3 \times 3 / 75 \%\end{array}$ & 8 Weeks & $\begin{array}{l}\text { CMJ } \\
\text { SJ }\end{array}$ & $\begin{array}{l}\text { Catch } \\
\uparrow \mathrm{SJ} \\
\mathrm{P}<0,001 \\
\uparrow \mathrm{CMJ} \\
\beta<0.001 \\
\quad \text { Pull } \\
\quad-\mathrm{SJ} \\
\uparrow \mathrm{CMJ} \\
p=0,04\end{array}$ \\
\hline $\begin{array}{l}\text { (Helland et al., } \\
\text { 2017) }\end{array}$ & $\begin{array}{c}\mathrm{n}=39 \\
\text { Atletas juveniles y adultos } \\
\text { alto rendimiento } \\
\text { (Bádminton } \\
\text { Ice Hockey Voleibol) }\end{array}$ & $\begin{array}{c}\text { Snatch } \\
\text { Clean+squat } \\
\text { EDH } \\
\text { HC HS }\end{array}$ & $\begin{array}{l}\text { 2/Weeks } \\
\& \\
\text { 3/Weeks }\end{array}$ & $\begin{array}{l}2 \text { a } 5 \text { ser } \\
3 \text { a } 5 \text { RM }\end{array}$ & 8 Weeks & $\begin{array}{l}\text { SJ, } \\
\text { CMJ } \\
\text { DJ }\end{array}$ & $\begin{array}{l}-\mathrm{SJ}, \\
-\mathrm{CMJ} \\
-\mathrm{DJ}\end{array}$ \\
\hline $\begin{array}{l}\text { (Hermassi et al., } \\
\text { 2019) }\end{array}$ & $\begin{array}{c}\mathrm{n}=22 \\
\text { Handbolistas } \\
1^{\circ} \text { Liga Nacional }\end{array}$ & $\begin{array}{c}\text { HSQ } \\
\text { Snatch } \\
\text { (alto) } \\
\text { Bench press } \\
\text { C \& J }\end{array}$ & 2/Weeks & $\begin{array}{l}55 \text { a } 75 \% \\
2 \text { a } 3 \text { ser } \\
6 \text { rep } \\
2 \text { a } 4 \text { ser } \\
6 \text { rep }\end{array}$ & 12 Weeks & $\begin{array}{c}\text { SJ } \\
\text { CMJ }\end{array}$ & $\begin{array}{c}\uparrow \mathrm{SJ} \\
\uparrow \mathrm{CMJ} \\
p<0,001\end{array}$ \\
\hline (Ince, 2019) & $\begin{array}{c}\text { n=34 } \\
\text { mujeres } \\
\text { Voleibolistas } \\
\text { Adolescentes }\end{array}$ & $\begin{array}{l}\text { Split HS } \\
\text { Split Clean } \\
\text { Split Jerk }\end{array}$ & 2/Weeks & $\begin{array}{c}70 \text { a } 85 \% \\
3 \text { a } 5 \text { ser } \\
5 \text { rep }\end{array}$ & 6 Weeks & $\begin{array}{l}\text { Spike } \\
\text { Jump } \\
\text { CMJ }\end{array}$ & $\begin{array}{c}\text { - CMJ } \\
\uparrow \text { Spike Jump } \\
\mathrm{d}=0,35 \\
\text { (Effect size) }\end{array}$ \\
\hline $\begin{array}{l}\text { (Keller et al., } \\
\text { 2018) }\end{array}$ & $\begin{array}{c}\mathrm{n}=12 \\
\text { Baloncesto } \\
\text { Adolescentes } \\
\text { Nivel regional }\end{array}$ & $\begin{array}{l}\mathrm{PC} \\
\mathrm{HP}\end{array}$ & 2/Weeks & $\begin{array}{l}4 \text { a } 6 \text { ser } \\
10 \text { a } 4 \text { rep }\end{array}$ & 4 Weeks & $\begin{array}{l}\text { CMJ } \\
\text { DJ } \\
\text { SLJ } \\
1 \mathrm{LLJ}\end{array}$ & $\begin{array}{c}\uparrow \mathrm{CMJ} \\
-\mathrm{DJ} \\
\uparrow \mathrm{SLJ} \\
\uparrow 1 \mathrm{LLJ} \\
p<0,05\end{array}$ \\
\hline $\begin{array}{l}\text { (Loturco et al., } \\
\text { 2016) }\end{array}$ & $\begin{array}{c}\mathrm{n}=17 \\
\text { Futbolistas élite } \\
\text { Sub } 20\end{array}$ & $\begin{array}{l}\text { GOLPP } \\
\text { o } \\
\text { GJSQ }\end{array}$ & 2/Weeks & $\begin{array}{c}\text { Optimal load } \\
6 \times 8 \\
6 \times 6 \\
6 \times 4\end{array}$ & 6 Weeks & $\begin{array}{c}\text { SJ } \\
\text { CMJ }\end{array}$ & $\begin{array}{l}-\mathrm{SJ} \\
-\mathrm{CMJ}\end{array}$ \\
\hline $\begin{array}{l}\text { (Oranchuk et al., } \\
\text { 2019) }\end{array}$ & $\begin{array}{c}\mathrm{n}=18 \\
\text { Nadadores } \\
\text { Universitarios }\end{array}$ & $\begin{array}{c}\text { HHP } \\
\text { o } \\
\text { JSQ } \\
\text { Trap-bar }\end{array}$ & 2/Weeks & $\begin{array}{c}70 \% \\
20 \% \\
\text { 3 a } 6 \text { ser } \\
2 \text { a } 5 \text { rep }\end{array}$ & 10 Weeks & $\begin{array}{c}\text { SJ } \\
\text { CM }\end{array}$ & $\begin{array}{c}\uparrow \mathrm{sJ}, \\
\uparrow \mathrm{CMJ} \\
p<0,05\end{array}$ \\
\hline \multicolumn{8}{|c|}{ 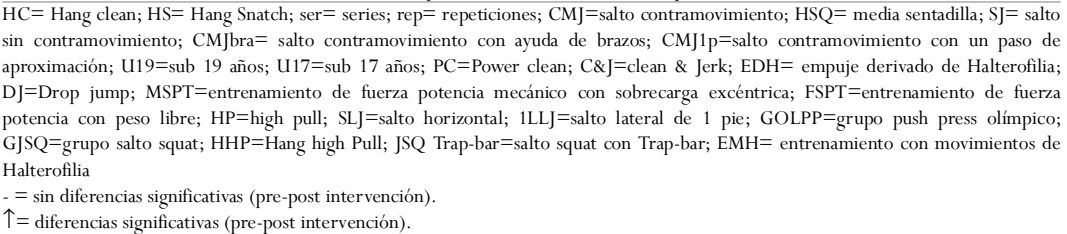 } \\
\hline
\end{tabular}


et al. (2016) ambas intervenciones basadas en Hang clean y Hang snatch mostraron mejoras significativas $(p \leq 0,01)$ en 40 yardas de sprint (36,5m). Así mismo, la intervención de Hermassi et al. (2019) mostró mejoras significativas $(p<0,001)$ en 5, 15, y 30 metros, al igual que el protocolo de EMH de estilo Split de Ince (2019) en 5 y 20 metros de sprint $(d=1,544 ; d=0,814)$.

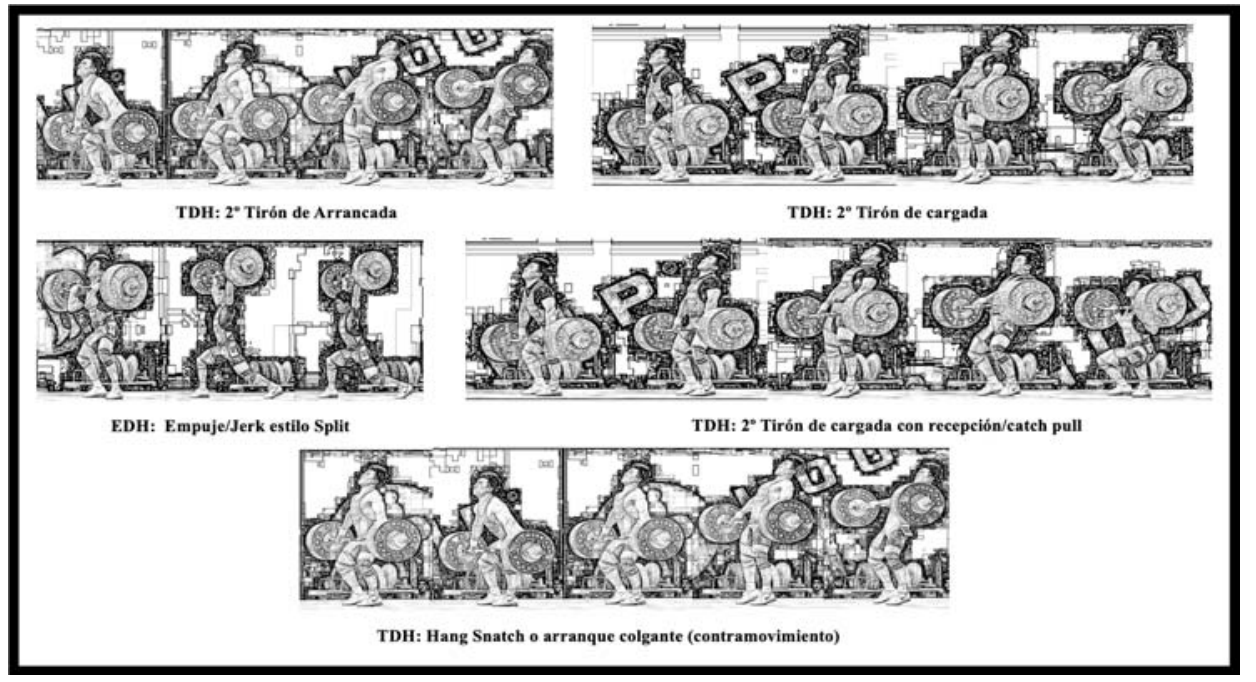

Figura 2. Fotogramas de ejercicios derivados principales de EMH

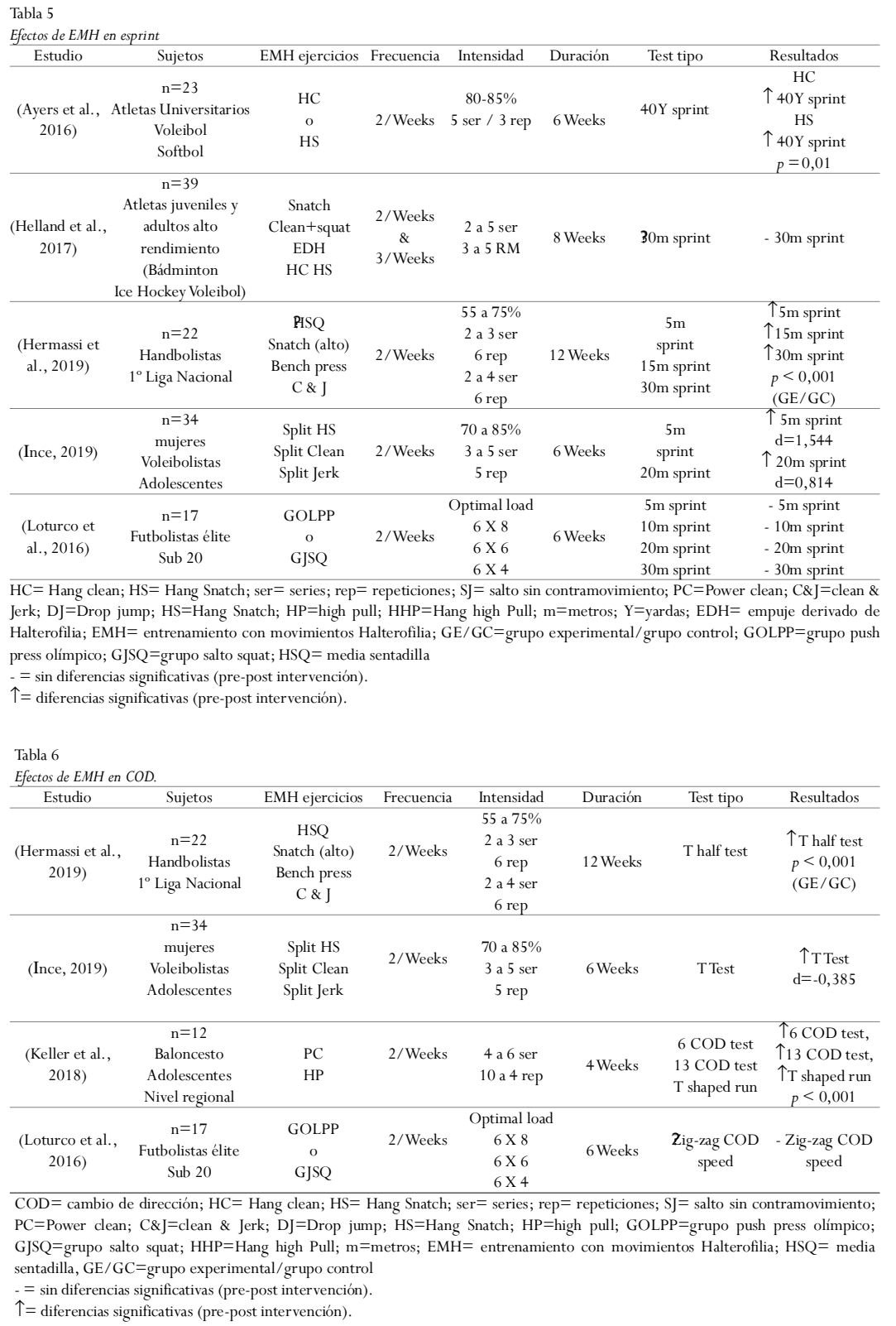

Dos de las cinco intervenciones no mostraron mejoras significativas, el protocolo de Helland et al. (2017) en 30 metros y el de Loturco et al. (2016) con evaluaciones de 5, 10, 20, y 30 metros de sprint.

Efectos del EMH en el rendimiento del cambio de dirección

En la Tabla 6 se muestra la información relacionada al rendimiento de COD. Las intervenciones de Hermassi et al. (2019) e Ince (2019), mostraron mejoras significativas en T half test $(p<0,001)$ y T test $(\mathrm{d}=$ $0,385)$. De la misma forma, el protocolo de Keller et al. (2018) mostró mejoras estadísticamente significativas en las pruebas de 6COD test, 13COD test, T shaped run. Únicamente la intervención de Loturco et al. (2016) no generó mejoras en COD, medida en el test Zig-zag change of direction speed.

\section{Discusión}

Según nuestro conocimiento, esta es la primera revisión sistemática que se centra solo en deportistas, ya sean profesionales, universitarios, de nivel nacional o regional y, además, es la única que reúne los resultados de las tres tareas motoras de salto, 
esprint y COD, las que son de alta trasferencia a la competencia. A continuación, se analizan las características de los EMH aplicados que mostraron efectos positivos en cada variable, y se discuten estos hallazgos contrastados con otros estudios. A su vez, para una mejor comprensión, en la Figura 2 se muestran los fotogramas que grafican los ejercicios de EMH más relevantes.

\section{EMH y el rendimiento en salto}

Se ha descrito que la capacidad de salto y la potencia se correlacionan con el rendimiento deportivo, por lo tanto mejorar la fuerza máxima y/o la velocidad aumentaría la producción de potencia, lo que llevaría a mejorar el desempeño en el juego (Darmiento, Galpin, \& Brown, 2012). El voleibol, uno de los deportes con más presencia en los artículos de esta revisión (Ayers et al., 2016; Helland et al., 2017; Ýnce, 2019), es una actividad con mucha demanda de la capacidad de salto, en consecuencia el rendimiento en este deporte depende altamente de la capacidad de realizar esfuerzos repetidos de potencia máxima o cercana a la máxima (Holmberg, 2013). El protocolo de Ince (2019), fue aplicado solo a jugadores de voleibol y se caracterizó por implementar ejercicios de EMH estilo Split (Split hang Snatch, Split clean, Split jerk). Estos movimientos se caracterizan por una potente separación anteroposterior de los miembros inferiores posterior a la triple extensión. Esta diferencia de ejecución técnica, en relación a los ejercicios derivados clásicos de EMH, permitiría explicar porque el CMJ no mostró mejoras con significancia estadística, y por otra parte, podría explicar las mejoras significativas en el Spike jump, un tipo de salto específico del voleibol que incluye desplazamiento horizontal y vertical que termina en golpes de balón que generan la mayor cantidad de puntos del juego (Holmberg, 2013).

Por otra parte, un estudio experimental de Comfort et al. (2018) aplicado a futbolistas, remadores, jugadores de hockey y deportistas de BMX, comparó los efectos del Power clean con recepción y sin recepción (TDH). Los resultados indicaron que ambos protocolos mejoraron el CMJ sin diferencias significativas entre el Power clean y su derivado sin recepción (Clean pull, TDH). Estos hallazgos confirman los resultados encontrados por Comfort, WIlliams, Suchomel, \& Lake, (2017), que explican que la fase de captura en el Power clean no proporciona ningún estímulo adicional en términos de fuerza concéntrica comparado con un Clean pull desde la rodillas ( sin captura / TDH). Sin embargo, a pesar de no haber diferencias en términos de fuerza y resultados, los TDH parecen ofrecer más beneficios al permitirle al deportista centrarse solamente en el segundo tirón y no acortar el recorrido (Schilling, 2016; Holmberg, 2013; Suchomel et al., 2015), ser más fáciles de aplicar técnicamente (Schilling, 2016; Hedrick, 2004; (Janz et al., 2008; Harbili \& Alptekin, 2014) y evitar lesiones por impacto en la fase de captura (Kulund et al., 1978; Stone et al., 1994; Suchomel \& Sato, 2013).

De particular interés son los resultados de protocolos de EMH que utilizaron derivados de tipo colgante (Hang clean, Hang Snatch, Hang high pull), los que resultaron en mejoras significativas en la capacidad de salto evaluada en CMJ y SJ (Ayers et al., 2016; Ciacci \& Bartolomei, 2018; Oranchuk et al., 2019; Hermassi et al.,2019). Estos resultados confirman los beneficios de los ejercicios colgantes, como el Hang power clean que mejora la potencia del tren inferior y la RFD (Ronai \& Scibek, 2016), el cual tiene una alta correlación con la capacidad de salto y esprint (Hori et al., 2008). Las razones que podrían explicar estos resultados tendrían relación con un menor recorrido en el que se debe aplicar fuerza comparado a otros ejercicios, y además, en que estos derivados de EMH aprovechan el ciclo estiramiento acortamiento (James, Suchomel, McMahon, Chavda, \& Comfort, 2020) al partir en posición colgante, lo cual aumenta la producción de potencia por la fase de estiramiento previa (Komi P.V \& Bosco C, 1978)(Anderson \& Pandy, 1993), sin embargo esto no ha sido demostrado directamente.

Por otra parte, las intervenciones de Ciacci \& Bartolomei (2018) e Ince (2019) evaluaron el nivel de rigidez (stiffness) de piernas, expresado en la relación de altura de saltos y tiempos de contacto. Esta variable mostró mejoras en stiffness de piernas en los grupos de U17 y U19, lo que puede explicar el aumento en SJ, CMJ, CMJbra, CMJ1paso. De la misma manera, el estudio de Ince (2019) también evidenció mejoras en el stiffness de los voleibolistas, lo que puede explicar las mejoras en el Spike Jump. Ambos estudios confirman la relación entre mayores niveles de rigidez y rendimientos más altos en movimientos rápidos que incluyen saltos y rebotes (Pruyn, Watsford, \& Murphy, 2014), y además, son coherentes con recomendaciones que plantean que el EMH impactan positivamente en la rigidez músculo tendinosa del tren inferior, adaptación que permite mayor acumulación de energía elástica y mayor generación de fuerza concéntrica en el despegue (Brazier, Bishop, Simons, Antrobus, Read \& Turner, 2014).

Interesantemente, los protocolos de EMH que no mostraron mejoras estadísticamente significativas en la 
capacidad de salto (Loturco et al., 2016; Helland et al., 2017), tienen como punto en común no aplicar o no dar predominancia a ejercicios de EMH centrados en el $2^{a}$ tirón, siendo esta acción técnica la más transferible al rendimiento deportivo (Suchomel et al., 2015; Schilling, 2016; Holmberg, 2013). Por ejemplo, en la intervención de Loturco et al. (2016), se aplicó el Push press que es un ejercicio EDH (Soriano et al., 2019) que no realiza $2^{a}$ tirón y que utiliza cargas más bajas, perdiendo los beneficios de los TDH que por mover cargas más pesadas y a alta velocidad mejoran el rendimiento atlético (Hori, Newton, Nosaka, \& Stone, 2005). Además en estudios posteriores, el mismo autor demostró mayor correlación entre Jump squat y capacidad de salto y esprint, comparado al Push press (Loturco et al., 2017). Por otro lado, a pesar de que Helland et al. (2017) incluye algunos ejercicios de EMH colgantes, los utiliza mezclándolos en el mismo entrenamiento con movimientos de halterofilia completos (Snatch, Clean \&Jerk), con EDH, e incluso algunos «complex» que combinan un ejercicio de potencia con uno de fuerza, lo cual evidencia la falta de enfoque en ejercicios con $2^{\circ}$ tirón.

\section{EMH y el rendimiento en esprint}

Los estudios de Ayers et al. (2016), Hermassi et al. (2019) e Ince (2019) mostraron mejoras significativas en la capacidad de esprint en distancias de 5, 15, 20, 30 metros y 40 yardas $(36,5 \mathrm{~m})$. Estos resultados comprueban el efecto positivo del EMH en la capacidad de esprint (Hedrick, 2018), y la razón por la que los entrenadores los utilizan en sus programas de fuerza y potencia (Bolger, Lyons, Harrison, \& Kenny, 2016; Chaouachi et al., 2014). Estas mejoras podrían explicarse debido a que el EMH desarrolla la producción de energía y produce más potencia de salida comparado a otros métodos, como por ejemplo el RTT, evidenciando que esta producción de potencia es más relevante en los deportes, en comparación a solo producir mayores niveles de fuerza (Hoffman et al., 2004).

Así mismo, El EMH desarrolla la capacidad de producir mayores niveles de fuerza y potencia vertical, y esta condición se relaciona con mejoras en el rendimiento de esprint (Hori et al., 2008). Además, esta alta producción de potencia se realiza en acciones multiarticulares de triple extensión que implican grandes volúmenes musculares, de hecho, un ejemplo de estas implicancias es el Power clean que utiliza más del $50 \%$ de la musculatura (Durck, 1986), y demanda un empuje agresivo que termina produciendo grandes niveles de fuerza de reacción contra el suelo, al igual que en un esprint (Hedrick, 2018).

Por otra parte, las intervenciones de Loturco et al. (2016) y Helland et al. (2017) no mostraron mejoras en la capacidad de esprint en 5, 10, 20, y 30 metros. En el caso de la intervención de Loturco et al. (2016), nuevamente la ausencia de ejercicios con $2^{\circ}$ tirón parece ser determinante para no producir efectos positivos en el esprint. Mientras que en el estudio de Helland et al. (2017), a pesar de existir algunos ejercicios enfocados en esta fase técnica, estos iban mezclados con otros tipos de ejercicios de EMH. La ausencia o falta de predominancia de ejercicios de EMH centrados en el $2^{\circ}$ tirón, parece ser determinante para la mejora del rendimiento, ya que en ellos se producen magnitudes más altas de fuerza máxima, RFD, velocidad y potencia (Suchomel et al., 2015). En el mismo sentido, esta característica del plan de entrenamiento parece ser más importante a la hora de influir en resultados positivos, ya que se utilizaron ejercicios de EMH colgantes, intensidades más elevadas (Soriano, Jiménez-Reyes, Rhea, \& Marín, 2015) ( $\geq 70 \%$ RM), e incluso se realizó un periodo de inducción técnica de dos semanas. Desde otra perspectiva, podemos explicar la ausencia de mejora basados en la teoría del vector de fuerza horizontal, que refiere a que los ejercicios que aplican fuerza en este sentido generan mejoras en el esprint (Contreras et al., 2017). Sin embargo, tampoco la teoría del vector horizontal y el rendimiento en sprint es determinante, ya que en el estudio de Los Arcos et al. (2014) si se encontraron mejoras significativas, tanto en entrenamientos que utilizaron ejercicios que aplicaban fuerza en el vector horizontal como vertical. Estos resultados abren nuevas preguntas para futuras investigaciones.

\section{EMH y el rendimiento en cambio de dirección}

Solo el estudio de Loturco et al. (2016) no mostró mejoras de significancia estadística en COD, especificamente en Zig-zag change of direction speed. En contra partida, los estudios de Hermassi et al. (2019), Ince (2019) y Keller et al. (2018) mostraron mejoras significativas en T half test, T test, 6COD test, 13COD test y $\mathrm{T}$ shaped run. Estos resultados se contraponen a las conclusiones de Brughelli et al. (2008), quien mostró evidencia de que los ejercicios de EMH no producen mejoras en la capacidad de COD. La revisión de 2008, explica que el entrenamiento de COD específico y los ejercicios que están en el vector horizontal son los que realmente generan mejoras en esta capacidad. Dicha aseveración encuentra más sustento en el estudio de Thomas, Dos'Santos, Comfort, \& Jones, (2018), que 
confirma que existe una asociación entre la fuerza unilateral y el rendimiento en COD, así como también, en los estudios de Meylan et al. (2009) y Lockie et al. (2014) que muestran alta correlación entre el rendimiento de los saltos unipodales horizontales y esta capacidad. Los resultados de nuestra revisión denotan la ausencia de investigaciones relacionadas directamente con el entrenamiento y la teoría del vector de fuerza en la capacidad de COD, pero aporta evidencia que demuestra que EMH si mejora la capacidad de COD en población deportista.

\section{Conclusiones}

Mediante esta revisión sistemática, podemos concluir que el EMH genera mejoras con significancia estadística en el rendimiento de salto, esprint y cambio de dirección aplicando distintos protocolos de ejercicios. Para garantizar estos efectos positivos, es recomendable usar ejercicios de Halterofilia derivados que se centren en el $2^{\mathrm{a}}$ tirón (TDH), ya que aquella fase es la que tiene mayor correspondencia con las tareas deportivas de triple extensión explosiva. Junto con ello, parece ser que TDH colgantes generan mejoras considerando que también aprovechan el ciclo de estiramiento-acortamiento en su ejecución de contramovimiento y presentan un recorrido más corto, lo que permite al deportista centrarse solo en el $2^{\circ}$ tirón y generar más potencia. Para futuras investigaciones, sería interesante comparar los efectos de los ejercicios TDH tipo colgantes con los derivados que parten desde la plataforma o de un apoyo más alto, para concluir sobre la implicancia de la ejecución con contramovimiento. Así como también, comparar los efectos en las habilidades atléticas de determinados tipos de ejercicios derivados del EMH con entrenamientos pliométricos, de resistencia tradicional, de sobrecarga excéntrica (MSPT) o kettelbells. Además, los distintos efectos encontrados en el rendimiento de COD y esprint, relacionados con ejercicios de fuerza aplicada en los vectores horizontales y verticales también abren una línea para futuras investigaciones.

\section{Aplicaciones prácticas}

La aplicación de este método de entrenamiento para el desarrollo de la potencia conlleva cierta dificultad técnica, debido a esto, es recomendable incluir en los protocolos de intervención fases de enseñanza metodológica, como se apreció en alguno de los estu- dios de la revisión. Sin embargo, cuando los atletas presentan problemas de movilidad, lesiones previas, dificultades en técnica de ejecución, o también se cuenta con poco tiempo para intervenir, se recomienda el uso de ejercicios derivados sin fase de captura (TDH) para evitar impactos y hacer uso eficiente del periodo de entrenamiento.

Se recomienda también, que si dentro de los objetivos del plan de entrenamiento se encuentra el desarrollo de la fuerza reactiva que implica esfuerzos del ciclo de estiramiento-acortamiento, se seleccionen dentro del programa ejercicios en sus variantes colgantes que tienen mayor similitud con tareas deportivas. En otro aspecto, si dentro del plan de entrenamiento se considera desarrollar capacidades de equilibrio y coordinación en contexto de potencia, los derivados de empujes sobre la cabeza (EDH) son una alternativa a utilizar.

Por último, en relación con la intensidad se recomienda planificar con intensidades $\geq 70 \%$ RM para el desarrollo eficaz de la expresión de fuerza-potencia necesaria para el rendimiento deportivo.

\section{Referencias}

Anderson, F. C., \& Pandy, M. G. (1993). Storage and utilization of elastic strain energy during jumping. Journal of Biomechanics, 26(12), 1413-1427. https://doi.org/ 10.1016/0021-9290(93)90092-S

Arabatzi, F., \& Kellis, E. (2012). Olympic weightlifting training causes different knee muscle--coactivation adaptations compared with traditional weight training. Journal OfStrength and Conditioning Research, 26(8), 2192-2201.

Ayers, J. L., Debeliso, M., Sevene, T. G., \& Adams, K. J. (2016). Hang cleans and hang snatches produce similar improvements in female collegiate athletes. Biology of Sport, 33(3), 251-256. https://doi.org/10.5604/ 20831862.1201814

Berton, R., Lixandrão, M. E., Pinto e Silva, C. M., \& Tricoli, V. (2018). Effects of weightlifting exercise, traditional resistance and plyometric training on countermovement jump performance: a meta-analysis. Journal of Sports Sciences, 36(18), 2038-2044. https://doi.org/10.1080/ 02640414.2018 .1434746

Bolger, R., Lyons, M., Harrison, A. J., \& Kenny, I. C. (2016). Coaching sprinting: Expert coaches' perception of resistance-based training. International Journal of Sports Science and Coaching, 11(5), 746-754. https://doi.org/ $10.1177 / 1747954116667113$

Bosco, C. (1994). La valoracion de la fuerza con el Test de Bosco (1 ${ }^{\text {a }}$ edicion). Barcelona: Paidotribo.

Bosco, C. , Luhtanen, P., \& Komi, P.V. (1983).A simple method for measurement of mechanical power in jumping. 
European Journal of Applied Physiology and Occupational Physiology, 50(2), 273-282. https://doi.org/10.1007/ BF00422166

Brazier, J., Bishop, C., Simons, C., Antrobus, M., Read, P. \& Turner, A. (2014). Lower extremity stiffness: Effects on performance and injury and implications for training, Strength \& Conditioning Journal, 36(5), 103-112. http:// doi.org/ 10.1519/SSC.0000000000000094

Brughelli, M., Cronin, J., Levin, G., \& Chaouachi, A. (2008). Understanding change of direction ability in sport: A review of resistance training studies. Sports Medicine, 38(12), 1045-1063. https://doi.org/10.2165/ 00007256-200838120-00007

Castro-Piñero, J., Ortega B., F., Artero G., E., Girela-Rejón, M. J., Mora, J., Sjostrom, M., \& R, R. J. (2010). Assessing muscular strength in youth: Usefulness of standing long jump as a general index of muscular fitness. Journal of Strength and Conditioning Research, 24(7), 1810-1817. Retrieved from https://pubmed.ncbi.nlm.nih.gov/ $20555277 /$

Channell, B.T., \& Barfield, J. P. (2008). Effect of olympic and traditional resistance training on vertical jump improvement in high school boys. Journal of Strength and Conditioning Research, 22(5), 1522-1527. https:// doi.org/10.1519/JSC.0b013e318181a3d0

Chaouachi, A., Hammami, R., Kaabi, S., Chamari, K., Drinkwater, E. J., \& Behm, D. (2014). Olympic weightlifting and plyometric training with children provides similar or greater performance improvements than traditional resistance training. Journal of Strength and Conditioning Research, 28(6), 1483-1496.

Chiu, L. Z. F., \& Salem, G. J. (2012). Potentiation of Vertical Jump Performance during a Snatch Pull Exercise Session. Journal of Applied Biomechanics, 28(6), 627-635. https: / / doi.org/10.1123/jab.28.6.627

Chiu, L. Z. F., \& Schilling, B. K. (2005). A primer on weightlifting: From sport to sports training. Strength and Conditioning Journal, 27(1), 42-48. https://doi.org/ $\begin{array}{lllllllllllll}1 & 0 & . & 1 & 5 & 1 & 9 & / & 1 & 5 & 3 & 3 & -\end{array}$ 4295(2005)027<0042:apowfs > 2.0.co;2

Ciacci, S. , \& Bartolomei, S. (2018). The effects of two different explosive strength training programs on vertical jump performance in basketball. Journal of Sports Medicine and Physical Fitness, 58(10), 1375-1382. https://doi.org/ 10.23736/S0022-4707.17.07316-9

Comfort, P., Allen, M., \& Graham-Smith, P. (2011a). Comparisons of peak ground reaction force and rate of force development during variations of the power clean. Journal of Strength and Conditioning Research, 25(5), 1235 1239. https://doi.org/10.1519/ JSC.0b013e3181d6dc0d

Comfort, P., Allen, M., \& Graham-Smith, P. (2011b). Kinetic comparisons during variations of the power clean. Journal of Strength and Conditioning Research, 25(12), 3269-3273. https: / / doi.org/10.1519/JSC.0b013e3182184dea

Comfort, P., Dos Santos, T., Christopher, T., ManMahon, J., \& Timothy, S. (2018). An investigation in to the effects of excluding the catch phase of the power clean on forcetime characteristics during isometric and dynamic tasks: an intervention study. Journal of Strength and Conditioning Research, 24(10), 1-14.

Comfort, P., Udall, R., \& Jones, P. A. (2012). The effect of Loading on kinematic and Kinetic variables during the midthigh clean pull. Journal OfStrength and Conditioning Research, 26(5), 1208-1214.

Comfort, P., WIlliams, R. W., Suchomel, T. J., \& Lake, J. P. (2017). A comparison of catch phase force-time characteristics during clean derivatives from the knee, 31(7), 1911-1918.

Contreras, B., Vigotsky, A. D., Schoenfeld, B. J., Beardsley, C., McMaster, D.T., Reyneke, J. H.T., \& Cronin, J. B. (2017). Effects of a Six-Week Hip Thrust vs. Front Squat Resistance Training Program on Performance in Adolescent Males: A Randomized Controlled Trial. Journal of Strength and Conditioning Research (Vol. 31). https://doi.org/ 10.1519/JSC.0000000000001510

Cormie, P., McGuigan, M., \& Newton, R. (2011). Developing Maximal Neuromuscular Power. Part 2 - Training Considerations for Improving Maximal Power Production. Sports Medicine, 41(2), 125-146.

Cronin, J., McNair, P. J., \& Marshall, R. N. (2003). The effects of bungy weight training on muscle function and functional performance. Journal of Sports Sciences, 21(1), 59-71. https: / / doi.org/10.1080/0264041031000071001

Cushion, E. J., Goodwin, J. E., \& Cleather, D. J. (2015). Relative intensity influences the degree of correspondence of jump squats and push jerks to countermovement jumps. Journal OfStrength and Conditioning Research, 30(5), 1255-1264.

Darmiento, A., Galpin, A. J., \& Brown, L. E. (2012). Vertical jump and power. Strength and Conditioning Journal, 34(6), 34-43. https://doi.org/10.1519/ SSC.0b013e3182752b25

de Morton, N. A. (2009). The PEDro scale is a valid measure of the methodological quality of clinical trials: a demographic study. Australian Journal of Physiotherapy, 55(2), 129-133. https://doi.org/10.1016/S00049514(09)70043-1

DeWeese, B. H., \& Scruggs, S. K. (2012). The countermovement shrug. Strength and Conditioning Journal, 34(5), 20-23. https://doi.org/10.1519/ SSC.0b013e $318262 \mathrm{f} 7 \mathrm{~d} 5$

DeWeese, B. H., Serrano, A. J., Scruggs, S. K., \& Burton, J. D. (2013). The midthigh pull: Proper application and progressions of a weightlifting movement derivative. Strength and Conditioning Journal, 35(6), 54 -58. https: / / doi.org/10.1519/SSC.0b013e318297c77b

DeWeese, B. H., Serrano, A. J., Scruggs, S. K., \& Sams, M. L. (2012). The clean pull and snatch pull: Proper technique 
for weightlifting movement derivatives. Strength and Conditioning Journal, 34(6), 82-86. https://doi.org/ 10.1519/SSC.0b013e31826f1023

DeWeese, B. H., Suchomel, T. J., Serrano, A. J., Burton, J. D., Scruggs, S. K., \& Taber, C. B. (2016). Pull from the knee: Proper technique and application. Strength and Conditioning Journal, 38(1), 79-85. https://doi.org/10.1519/ SSC.0000000000000194

Durck, C. (1986). Squat and power clean relationship to sprint training. National Strength and Conditioning Association, 8(6), 40-41.

Ebben, W. P., Carrol, R. M., \& Simenz, C. J. (2004). Strength and conditioning practices of National Hockey League strength and conditioning coaches. Journal of Strength and Conditioning Research, 18(4), 889-897. https://doi.org/ $10.1519 / 14133.1$

Ebben,W. P., Hintz, M. J., \& Simenz, C. J. (2005). Strength and conditioning practices of Major League Baseball strength and conditioning coaches. Journal of Strength and Conditioning Research, 19(3), 538-546. https://doi.org/ 10.1519/R-15464.1

Gabbett, T., Georgieff, B., Anderson, S., Cotton, B., Savovic, D., \& Nicholson, L. (2006). Changes in skill and physical fitness following training in talent-identified volleyball players. Journal of Strength and Conditioning Research, 20(1), 29-35. https://doi.org/10.1519/R-16814.1

García-Chaves, D. C., Corredor-Serrano, L. F., \& ArboledaFranco, S. A. (2020). Relación entre potencia muscular, rendimiento físico y competitivo en jugadores de baloncesto. Retos, 41, 191-198. https://doi.org/10.47197/ retos.v0i41.82748

Gastin, P. B. (2001). Energy system interaction and relative contribution during maximal exercise. Sports Medicine, 31(10), 725-741. https://doi.org/10.2165/ 00007256-200131100-00003

Hackett, D., Davies, T., Soomro, N., \& Halaki, M. (2016). Olympic weightlifting training improves vertical jump height in sportspeople: A systematic review with metaanalysis. British Journal of Sports Medicine, 50(14), 865872. https: / /doi.org/10.1136/bjsports-2015-094951

Harbili, E., \& Alptekin, A. (2014). Comparative kinematic analysis of the snatch lifts in elite male adolescent weightlifters. Journal of Sports Science and Medicine, 13(2), 417-422.

Haugen, T., Seiler, S., Sandbakk, Ø., \&Tønnessen, E. (2019). TheTraining and Development of Elite Sprint Performance: an Integration of Scientific and Best Practice Literature. Sports Medicine - Open, 5(1). https://doi.org/10.1186/ s40798-019-0221-0

Hedrick,A. (2004). Teaching the clean. Strength and Conditioning Journal, 26(4), 70-72. https://doi.org/10.1519/ 00126548-200408000-00014

Hedrick, A. (2018). Weightlifting Movements and Sprint Performance. Strength and Conditioning Journal, 40(1), 92-97. https: / /doi.org/10.1519/SSC.0000000000000357

Helland, C., Hole, E., Iversen, E., Olsson, M. C., Seynnes, O., Solberg, P.A., \& Paulsen, G. (2017).Training strategies to improve muscle power: Is olympic-style weightlifting relevant? Medicine and Science in Sports and Exercise, 49(4), 736-745. https://doi.org/10.1249/ MSS.0000000000001145

Herbert, R., Moseley,A., Sherrington, C., \& Maher, C. (2000). Escala PEDro-Español. Centre for Evidence-Based Physiotherapy (CEBP), 86(1), 55. https://doi.org/ 10.1016/S0031-9406(05)61357-0

Hermassi, S., Schwesig, R., Aloui, G., Shephard, R. J., \& Chelly, M. S. (2019). Effects of Short-Term In-SeasonWeightlifting Training on the Muscle Strength, Peak Power, Sprint Performance, and Ball-Throwing Velocity of Male Handball Players. Journal of Strength and Conditioning Research, 33(12), 3309-3321. https://doi.org/10.1519/ JSC.0000000000003068

Hoffman, J. R., Cooper, J., Wendell, M., \& Kang, J. (2004). Comparison of olympic vs. traditional power lifting training programs in football players. Journal of Strength and Conditioning Research, 18(1), 129-135. https: / / doi.org/ 10.1519/00124278-200402000-00019

Holmberg, P. M. (2013). Weightlifting to improve volleyball performance. Strength and Conditioning Journal, 35(2), 7988. https://doi.org/10.1519/SSC.0b013e3182889f47 Hori, N., Newton, R. U., Andrews, W. A., Kawamori, N., Mcguigan, M. R., \& Nosaka, K. (2008). Does performance of hang power clean differentiate performance of jumping, sprinting, and changing of direction? Journal of Strength and Conditioning Research, 22(2), 412-418. https: / /doi.org/10.1519/JSC.0b013e318166052b

Hori, N., Newton, R. U., Nosaka, K., \& Stone, M. H. (2005). Weightlifting Exercises Enhance Athletic Performance:That Requires High-Load Speed Strength. Strength and Conditioning Journal, 27(4), 50-55.

Hutton, B., Catalá-López, F., \& Moher, D. (2016). La extensión de la declaración PRISMA para revisiones sistemáticas que incorporan metaanálisis en red: PRISMA-NMA. Medicina Clinica, 147(6), 262-266. https://doi.org/ 10.1016/j.medcli.2016.02.025

Ýnce, Ý. (2019). Effects of split style olympic weightlifting training on leg stiffness vertical jump change of direction and sprint in collegiate volleyball players. Universal Journal of Educational Research, 7(1). https://doi.org/10.13189/ ujer.2019.070104

International Weightlifting Federeation. (2018). IWF-2018 Technical and competition rules \& regulations. (Á. Attila, R. Chinen, S. Coffa, Lassen.Moira, M. Lencsér, \&A. NémethMora, Eds.) (Dr. Tamás). Budapest 1146, Istvánmezei út 1-3. Hungary:The InternationalWeightlifting Federation. James, L. P., Suchomel, T. J., McMahon, J. J., Chavda, S., \& Comfort, P. (2020). Effect of OnsetThreshold on Kinetic and Kinematic Variables of a Weightlifting Derivative 
Containing a First and Second Pull. Journal of Strength and Conditioning Research, 34(2), 298-307. https://doi.org/ 10.1519/JSC.0000000000003316

Janz, J., Dietz, C., \& Malone, M. (2008).Training explosiveness: Weightlifting and beyond. Strength and Conditioning Journal, 30(6), 14-22. https://doi.org/10.1519/ SSC.0b013e31818e 2f13

Kawamori, N., \& Haff, G. G. (2004). The Optimal Training Load for the Development of Muscular Power. The Journal of Strength and Conditioning, 18(3), 675-684. https:// doi.org/10.1519/1533-4287(2004)18<675

Keller, S., Koob, A., Corak, D., von Schöning,V., \& Born, D. P. (2018). How to Improve Change-of-Direction Speed in JuniorTeam Sport Athletes-Horizontal, Vertical, Maximal, or Explosive Strength Training? Journal of Strength and Conditioning Research, 34(2), 473-482. https: / / doi.org/ 10.1519/JSC.0000000000002814

Komi P. V, \& Bosco C. (1978). Utilization of stored elastic energy in leg extensor muscles by men and women. $\mathrm{Me}$ dicine and Science in Sports. Retrieved from http:/ / e.guigon.free.fr/rsc/article/KomiBosco78.pdf

Kulund, D. N., Dewey, J. B., Brubaker, C. E., \& Roberts, J. R. (1978). OlympicWeight-Lifting Injuries. The Physician and Sportsmedicine, 6(11), 111-119. https://doi.org/ 10.1080/00913847.1978.11948407

Lockie, R. G., Callaghan, S. J., Berry, S. P., Cooke, E., Jordan, C.A., Luczo, T. M., \& Jeffriess, M. D. (2014). Relationship between unilateral jumping ability and asymmetry on multidirectional speed in team-sport athletes. Journal of Strength and Conditioning Research, 28(12), 3557-3566.

Los Arcos,A., Yanci, J., Mendiguchia, J., Salinero, J. J., Brughelli, M., \& Castagna, C. (2014). Short-term training effects of vertically and horizontally oriented exercises on neuromuscular performance in professional soccer players. International Journal of Sports Physiology and Performance, 9(3), 480-488. https://doi.org/10.1123/ IJSPP.2013-0063

Loturco, I., Kobal, R., Maldonado, T., Piazzi,A. F., Bottino, A., Kitamura, K., ... Nakamura, F.Y. (2017). Jump Squat is More Related to Sprinting and Jumping Abilities than Olympic Push Press. International Journal of Sports Medicine, 38(8), 604 612. https://doi.org/10.1055/s-00351565201

Loturco, Irineu, Pereira, L.A., Kobal, R., Maldonado, T., Piazzi, A. F., Bottino, A., ... Nakamura, F.Y. (2016). Improving sprint performance in soccer: Effectiveness of jump squat and olympic push press exercises. PLoS ONE, 11(4). https: / / doi.org/10.1371/journal.pone.0153958

Maffiuletti, N. A., Aagaard, P., Blazevich,A. J., Folland, J., Tillin, N., \& Duchateau, J. (2016). Rate of force development: physiological and methodological considerations. European Journal of Applied Physiology, 116(6), 1091-1116. https: / / doi.org/10.1007/s00421-016-3346-6

Maloney, S. J., Turner,A. N., \& Fletcher, I. M. (2014). Ballistic
Exercise as a Pre-Activation Stimulus: A Review of the Literature and Practical Applications. Sports Medicine, 44(10), 1347-1359. https://doi.org/10.1007/ s40279-014-0214-6

Mcbride, J. M., Triplett-Mcbride,T., Davie,A., \& Newton, R. U. (2002). The effect of heavy- vs. light-load jump squats on the development of strength, power, and speed. Journal of Strength and Conditioning Research, 16(1), 75-82. https: / / d o i.org/ 10 . $1519 / 4533$ 4287(2002)016<0075:TEOHVL > 2.0.CO;2

Methley, A. M., Campbell, S., Chew-Graham, C., McNally, R., \& Cheraghi-Sohi, S. (2014). PICO, PICOS and SPIDER:A comparison study of specificity and sensitivity in three search tools for qualitative systematic reviews. BMC Health Services Research, 14(1). https://doi.org/ 10.1186/s12913-014-0579-0

Meylan, C., McMaster,T., Cronin, J., Mohammad, N. I., Rogers, C., \& Deklerk, M. (2009). Single-leg lateral, horizontal, and vertical jump assessment: Reliability, interrelationships, and ability to predict sprint and changeof-direction performance. Journal of Strength and Conditioning Research, 23(4), 1140-1147. https:// doi.org/10.1519/JSC.0b013e318190f9c2

Miras Moreno, S. (2019). La altura del salto en contramovimiento como instrumento de control de la fatiga neuromuscular. Revisión sistemática. Retos, 37, 820826. https://doi.org/10.47197/retos.v37i37.73302

Mooney, M., Brien, B., Cormack, S., Coutts, A., Berry, J., \& Young, W. (2011). The relationship between physical capacity and match performance in elite Australian football: A mediation approach. Journal of Science and Medicine in Sport, 14(5), 447-452. https://doi.org/10.1016/ j.jsams.2011.03.010

Oranchuk, D. J., Robinson, T. L., Switaj, Z. J., \& Drinkwater, E. J. (2019). Comparison of the Hang High Pull and Loaded Jump Squat for the Development of Vertical Jump and Isometric Force-Time Characteristics. Journal of Strength and Conditioning Research, 33(1), 17-24. https: / /doi.org/10.1519/JSC.0000000000001941

Otto, W. H., Coburn, J. W., Brown, L. E., \& Spiering, B. A. (2012). Effects of weightlifting vs. kettlebell training on vertical jump,strength, and body composition. Journal OfStrength and Conditioning Research, 26(5), 1199-1202.

Pruyn, E. C., Watsford, M., \& Murphy, A. (2014). The relationship between lower-body stiffness and dynamic performance. Applied Physiology, Nutrition and Metabolism, 39(10), 1144-1150. https://doi.org/10.1139/apnm2014-0063

Rodríguez-Rosell, D., Pareja-Blanco, F., Aagaard, P., \& González-Badillo, J. J. (2018). Physiological and methodological aspects of rate of force development assessment in human skeletal muscle. Clinical Physiology and Functional Imaging, 38(5), 743-762. https: / / doi.org/ $10.1111 / \mathrm{cpf} .12495$ 
Ronai, P., \& Scibek, E. (2016).The Hang Power Clean. ACSM’s Health and Fitness Journal, 20(5), 50-55. https: / / doi.org/ 10.1249/FIT.0000000000000240

Sattler,T., Sekulic, D., Hadzic, V., Uljevic, O., \& Dervisevic, E. (2012). Vertical jumping tests in volleyball: Reliability, validity, and playing-position specifics. Journal of Strength and Conditioning Research, 26(6), 1532-1538. https:// doi.org/10.1519/JSC.0b013e318234e838,

Scanlan, A., Dascombe, B., \& Reaburn, P. (2011).A comparison of the activity demands of elite and sub-elite Australian men's basketball competition. Journal of Sports Sciences, 29(11), 1153-1160. https://doi.org/10.1080/ 02640414.2011.582509

Schilling, J. F. (2016). Weightlifting Exercises for LowerExtremity Power. ACSM1/4S Health \& Fitness Journal, 20(3), 16-21. fit.0000000000000201

Simenz, C. J., Dugan, C. A., \& Ebben, W. P. (2005). Strength and Conditioning Practices of National Basketball Association Strength and Conditioning Coaches. Journal of Strength and Conditioning Research, 19(3), 495-504. https:// doi.org/10.1519/00124278-20050800000003

Soriano, Marco A., Jiménez-Reyes, P., Rhea, M. R., \& Marín, P. J. (2015). The Optimal Load for Maximal Power Production During Lower-Body Resistance Exercises: A Meta-Analysis. Sports Medicine, 45(8), 1191-1205. https:/ /doi.org/10.1007/s40279-015-0341-8

Soriano, Marcos A., Suchomel, T. J., \& Comfort, P. (2019). Weightlifting Overhead Pressing Derivatives: A Review of the Literature. Sports Medicine (Auckland, N.Z.), 49(6), 867-885. https://doi.org/10.1007/s40279-01901096-8

Stone, M. H., Fry,A. C., \& Ritchie, M. (1994). Injury potential and safety aspects of weightlifting movements. Strength and Conditioning Journal, 16(3), 15-21.

Suchomel, T. J., Comfort, P., \& Stone, M. H. (2015). Weightlifting Pulling Derivatives: Rationale for Implementation and Application. Sports Medicine, 45(6), 823-839. https://doi.org/10.1007/s40279-0150314-y

Suchomel, T. J., DeWeese, B. H., Beckham, G. K., Serrano, A. J., \& French, S. M. (2014). The hang high pull: A progressive exercise into weightlifting derivatives. Strength and Conditioning Journal, 36(6), 79-83. https: / / doi.org/ 10.1519/SSC.0000000000000089

Suchomel, T. J., DeWeese, B. H., Beckham, G. K., Serrano, A. J., \& Sole, C. J. (2014). The jump shrug: A Progressive exercise Into weightlifting derivatives. Strength and Conditioning Journal, 36(3), 43-47. https://doi.org/ $10.1519 /$ ssc.0000000000000064

Suchomel, T. J., Nimphius, S., Bellon, C. R., \& Stone, M. H. (2018). The Importance of Muscular Strength: Training Considerations. Sports Medicine. https://doi.org/ 10.1007/s40279-018-0862-z
Suchomel, T. J., Nimphius, S., \& Stone, M. H. (2016). The Importance of Muscular Strength in Athletic Performance. Sports Medicine. https://doi.org/10.1007/s40279016-0486-0

Suchomel,T. J., \& Sato, K. (2013). Baseball ResistanceTraining: Should Power Clean Variations Be Incorporated? Journal of Athletic Enhancement, 02(02). https://doi.org/ 10.4172/2324-9080.1000112

Suchomel, T. J., \& Sole, C. J. (2017). Power-Time Curve Comparison between Weightlifting Derivatives. Journal of Sports Science \& Medicine, 16(3), 407-413. Retrieved from https: / / www.ncbi.nlm.nih.gov/pmc/articles / PMC5592293/

Suchomel, T. J., Wright, G. A., Kernozek, T.W., \& Kline, D. E. (2014). Kinetic comparison of the power development between power clean variations. Journal of Strength and Conditioning Research, 28(2), 350-360. https:/ / doi.org/ 10.1519/JSC.0b013e31829a36a3

Sullivan, C., Bilsborough, J. C., Cianciosi, M., Hocking, J., Cordy, J., \& Coutts, A. J. (2014). Match score affects activity profile and skill performance in professional Australian Football players. Journal of Science and Medicine in Sport, 17(3), 326-331. https://doi.org/10.1016/ j.jsams.2013.05.001

Sweeting, A. J., Cormack, S. J., Morgan, S., \& Aughey, R. J. (2017). When is a sprint a sprint? A review of the analysis of team-sport athlete activity profile. Frontiers in Physiology, 8(432), 1-12. https://doi.org/10.3389/ fphys.2017.00432

Teo, S.Y. M., Newton, M. J., Newton, R. U., Dempsey, A. R., \& Fairchild, T. J. (2016). Comparing the effectiveness of a short-term vertical jump vs. Weightlifting program on athletic power development. Journal of Strength and Conditioning Research, 30(10), 2741-2748. https:// doi.org/10.1519/JSC.0000000000001379

Thomas, C., Dos'Santos, T., Comfort, P., \& Jones, P. (2018). Relationships between Unilateral Muscle Strength Qualities and Change of Direction in Adolescent TeamSport Athletes. Sports, 6(3), 83. https://doi.org/ $10.3390 /$ sports6030083

Tricoli,V., Lamas, L., Carnevale, R., \& Ugrinowitsch, C. (2005). Short-term effects on lower-body functional power development: Weightlifting vs. vertical jump training programs. Journal of Strength and Conditioning Research, 19(2), 433-437. https://doi.org/10.1519/R-14083.1

Trueba-Gómez, R., \& Estrada-Lorenzo, J. M. (2010). La base de datos PubMed y la búsqueda de información científica. Seminarios de La Fundacion Espanola de Reumatologia, 11(2), 49-63.https: / / doi.org/10.1016/ j.semreu.2010.02.005 